\title{
A Improve Robust Watermarking Algorithm for Binary Images
}

\author{
Zhang Lei \\ Electric and Automation Engineering Department \\ Shandong Shengli Vocational College \\ Dongying, China \\ e-mail: diangongzl@163.com
}

\begin{abstract}
A improve robust watermarking algorithm is proposed for binary images. The primary focus of the proposed algorithm lies in finding more flippable pixels and enhancing the robustness of watermarking. Based on Haar Wavelet Transform, the more flippable pixels was found not only in the neighbourhood but also in the Wavelet Transform domain. The embedding mechanism based on dynamic step size was proposed for enhancing the robustness of watermarking. And the experiment results and analyses demonstrate that proposed algorithm can find more flippable pixels, and is has better in robustness and invisibility.
\end{abstract}

Keywords- Binary images; Digital watermarking; Haar wavelet transform; Data hiding

\section{INTRODUCTION}

The watermarking for binary image is more difficult than that of colour or gray images in which the pixels take on a wide range of values. It is very challenging to undetectably embed information into a binary image. In recent years, with the development of binary images watermarking, there were a few methods being proposed, such as spatial domain [1] [2], wavelet transform domain [3] [4] and specific format document [5]. More than that, some of them put forward many good suggestions on enhancing capacity [6], developing robustness [7] and improving security [8] for binary images.

$\mathrm{Wu}$ [1] referred a watermarking algorithm for authentication. It constructs a predefined flippability lookup table and removes some most flappable pixels to embed one bit in one block, observing the smoothness and connectivity. However, the flippablity score of each pixel is isolated, so some pixels that can be flipped are neglected. Kwon [3] proposed another embedding mechanism based on BWT (Binary Wavelet Transform). It removes pixels in the sub image of the high pass filter. However, the sub image of the high pass filter is only a quarter of the original in size, which limits the capacity.

In this paper, a novel algorithm is proposed to embed data in binary images. More flippable pixels are found, aimed at enhancing the capacity. The flippablity of a pixel is determined not only by its neighborhood, but also by the Wavelet Transform domain. Then, we partition the binary image into the no overlapping blocks, and use the dynamic step size to compute the watermarking data, instead of a constant. It improves the robustness of the watermarking. The experiments show the advantage of proposed algorithm, compared with the traditional method.
This paper is organized as follows. The proposed exact watermarking algorithm is described in Section 2. The robustness analysis of algorithm is described in Section 3. The experimental results are revealed in Section 4 and the conclusion of this paper is shown in Section 5.

\section{WATERMARK EMBEDDING ALGORITHM}

\section{A. Method of Finding Flippable Pixels Based on Haar Wavelet Transform}

Conceptually, Haar Wavelet is simple because it is constructed from a square wave:

$$
\begin{aligned}
& \cdot \phi_{L}(n)=\left\{\begin{array}{cl}
\frac{1}{\sqrt{2}} & n=2 k, 2 k+1 \\
0 & \text { elsewhere }
\end{array}\right. \\
& \phi_{H}(n)=\left\{\begin{array}{cl}
\frac{1}{\sqrt{2}} & n=2 k \\
-\frac{1}{\sqrt{2}} & n=2 k+1 \\
0 & \text { elsewhere }
\end{array}\right.
\end{aligned}
$$

Haar Wavelet computation is fast since it only contains two coefficients and does not need a temporary array for multi-level transformation [9]. Thus, after undergoing the Wavelet Transform, each pixel will be used once and no pixel overlapping during the computation [10].

The flippablity approach that $\mathrm{Wu}$ referred takes the human visual model for binary images. It is fit to watermarking embedded scheme for binary images. More details about this approach are referred to [1]. However, it neglects some flippable pixels. So, we find these pixels with the help of Haar Wavelet Transform. Before the measurement by the $3 \times 3$ window, the original binary image, such as Fig. 1, goes through the Haar Wavelet Transform. Then, four band images can be obtained (i.e. LL, LH, HL and $\mathrm{HH}$ ), shown in Fig. 2. The first band called as approximation represents the transform filtered with a low pass filter and compressed to half. It reveals the smoothness and connectivity part of the original. The other three bands (LH, HL and $\mathrm{HH})$ are called as details, where high pass filter is applied. These bands contain the edge properties of 
different directions. The size of each band is also compressed to half. We can use these properties to find the flippable pixels in the binary images. According to the analyses above, the flip rules is described as follows:

- If the pixel in the original image is 0 , and the corresponding coefficient is lager than 0 in the LL band; give the pixel a filppable score. The lager the coefficient is, the higher the score. Otherwise, if the pixel in the original image is 1 , and the corresponding coefficient is less than 1 in the LL band; give the pixel a score, too. The lager the coefficient is, the lower the score.

- Give the pixel mapped in the LH, HL and HH a fippable score. The larger the coefficient is, the higher the score.

- The lowest score is assigned to uniformly white or black regions as well as to the isolated single white or black pixels.

- If the number of transitions along horizontal or vertical direction is zero, assign zero as a final score for the current pixel. Otherwise, assign to the pixel a base score $S_{B}$ and proceed to the next rule.

- If the number of transitions along diagonal or antidiagonal direction is zero, reduce the score. Otherwise, if the minimum number of transition points along any one of the four directions is below a given threshold $T_{1}$, reduce the score by a smaller amount.

- If flipping the centre pixel does not change the number of transition points, increase the score. Otherwise, decrease the score.

- If flipping changes the number of black clusters or white clusters, reduce the score.

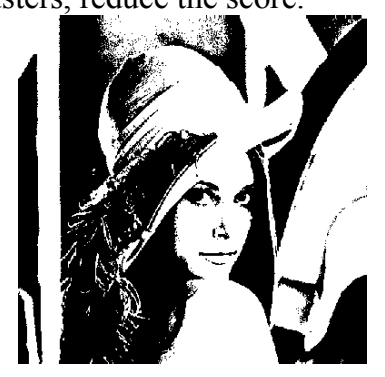

Figure 1. Original images

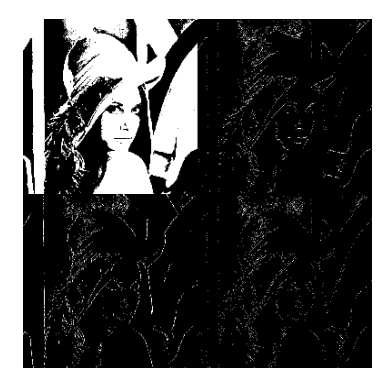

Figure 2. Wavelet wransform coefficients

\section{B. Embedding Mechanism}

The mechanism that $\mathrm{Wu}$ [1] referred is based on the partitioned blocks. However, the robustness against kinds of attack is quite limited. It is necessary to improve the algorithm to develop the robustness of the watermarking. Therefore, a novel mechanism is proposed to enhance the robustness of binary watermarking.

The embedding steps are described as follows:

- It is supposed that $w_{1}, w_{2}, \ldots w_{n}$ are the watermarking data;

- According to the flippablity approach that A referred, give each pixel a flippable score;

- Use shuffling to equalize the uneven embedding capacity and partition the binary image into blocks: $A_{1}, A_{2} \ldots A_{L}$, here $L>n$;

- $\quad$ Divide every block into embedded area $U_{i}$ and level area $V_{i}$, and compute the number of black pixel in two areas: $u_{1}, u_{2}, \ldots, u_{L}$ and $v_{1}, v_{2}, \ldots, v_{L}$. The number of black pixels in every block: $m_{i}=u_{i}+v_{i}$;

- If $\mathrm{K}$ is the dynamic step size, make $\mathrm{K}$ be in the direction proportion to the number of black pixel, namely $K_{i}=\alpha m_{i}$, where, $\alpha$ is the coefficient of adjustment;

- Compute the number of black pixel in the embedded area of $A_{i}$. If

$$
\left(\frac{u_{i}}{K_{i}}\right) \bmod 2=w_{i}^{\prime}
$$

- the pixels remain invariable, or flip $\Delta_{i}$, according to the watermarking information $w_{i}{ }^{\prime}$, let

$$
\left(\frac{u_{i}+\Delta_{i}}{K_{i}}\right) \bmod 2=w_{i}{ }^{\prime}
$$

- According to the number of flipped pixel in the embedded area, flip opposite color pixels in the level area, the number of flipped pixel in the embedded area $\Delta_{i}$ equals that in the level area $\Lambda_{i}$, namely $\Delta_{i}=\Lambda_{i}$;

Watermarking extraction is performed in a similar way to embedding.

\section{ROBUSTNESS ANALYSIS}

The most frequent factors affecting the watermarking are noise and scaling transformer. The scaling transformer is a kind of blind attack on watermarked binary images. Gaussian noise, salt and pepper noise and JPEG compression degrade images in the transmission process.

The attacks on the watermarked binary images can be described as the procedure of convolution:

$$
F_{w}(x)=p(x)^{*} F(x) .
$$


Where, $\mathrm{p}$ is the level of attacks, $\mathrm{x}$ is the pixels, $F_{w}$ is the detected image, and $F$ is the watermarked image. It is supposed that $\mathrm{I}$ is the image block, $I_{u}$ is the embedded area, and $I_{v}$ is the level area:

$$
\int_{I} F_{w}(x) d x=\int p(x) d x \int_{I} F(x) d x
$$

Because the step size $K_{i}$ is in the direction proportion to the number of black pixel, if the images are attacked, the change ratio of $K_{i}$ is the direction proportion to that of $m_{i}$, namely:

$$
\begin{aligned}
w_{t i}^{\prime} & =\frac{\int_{I_{u}} F_{w}(x) d x}{\alpha \int_{I} F_{w}(x) d x} \\
& =\frac{\int p(x) d x \int_{I_{u}} F(x) d x}{\alpha \int p(x) d x \int_{I} F(x) d x} \\
& =\frac{\int_{I_{u}} F(x) d x}{\alpha \int_{I} F(x) d x} \\
& =w_{i}
\end{aligned}
$$

According to the above analyses, the attacks on the binary images can be reduced, with the help of dynamic step.

\section{EXPERIMENTAL RESULTS}

We have tested our algorithm on a wide range of binary images. The results are similar. We have removed all black and white pixels respectively in the original binary images. Fig. 3 is the images where all white flippable pixels have been flipped to black, which is indistinguishable from Fig.1.

We also have tested the robustness against the common attacks. The experiment results are revealed in Fig.4. The red dots show the probability of error in proposed algorithm. Since the number of pixel is integer, if the scaling ratio is integer, the probability of error is zero; if not, the error probably exists. The results of noise attacking experiments show that the effect of Gaussian noise is less than salt and pepper noise. Because salt and pepper noise changes the number of black pixel, it has a greater influence on correct detection of watermarking. Moreover, the ratio of JPEG compression is low in binary images. Therefore, the effect of it on binary images is not too much. The result of the experiment shows that the proposed algorithm can reduce the effect of common attacks.

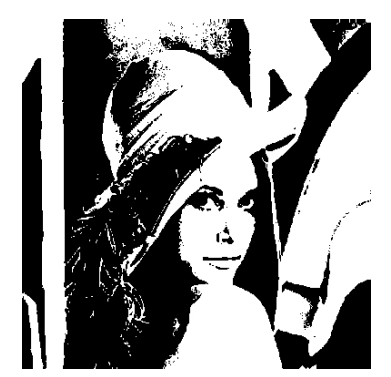

Figure 3. All white flippable pixels flipped to black

TABLE I. EXPERIENCE RESULTS

\begin{tabular}{|c|c|c|}
\hline \multicolumn{2}{|c|}{ Sample image } & Picture \\
\hline \multicolumn{2}{|c|}{ Image size } & $384 \times 384$ \\
\hline Number of black pixels & 70590 \\
\hline $\begin{array}{c}\text { Number of Black } \\
\text { flappable pixels }\end{array}$ & Wu's algorithm & 1909 \\
\cline { 2 - 3 } & Proposed algorithm & 2502 \\
\hline \multirow{2}{*}{$\begin{array}{c}\text { Number of White } \\
\text { flappable pixels }\end{array}$} & Wu's algorithm & 1809 \\
\cline { 2 - 3 } & Proposed algorithm & 2567 \\
\hline \multirow{2}{ELD_{\text{total}}}{$* N$} & Wu's algorithm & 2.5217 \\
\cline { 2 - 3 }$N$ & Proposed algorithm & 2.4063 \\
\hline
\end{tabular}

For check the proposed method against traditional method, several testing was made. The result is shown in Table 1 and Fig. 4. Recently, several distortion measurements have been proposed for binary images. The most common measurement is ELDM. It considers not only how many pixels are changed, but also where the pixels are changed and how the flipping affects the overall shape formed by the edge line. We embed the same watermarking in the same original with different method. Then, use the ELDM to measure the visual distortion. Because we consider not only the connectivity and the smoothness, but also the edge property of different directions with the help of Haar Wavelet Transform, the invisibility is better than in common method. The green dots in Fig. 4 show the probability of error in common algorithm. It can be seen that our algorithm can find more flippable pixels and is better in robustness with better invisibility.

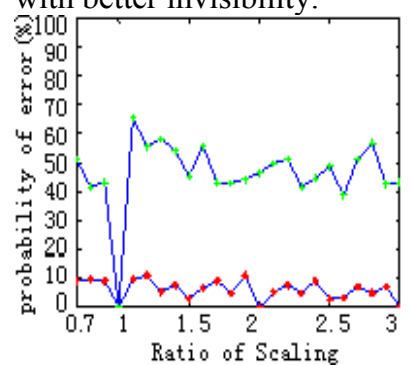

(a)

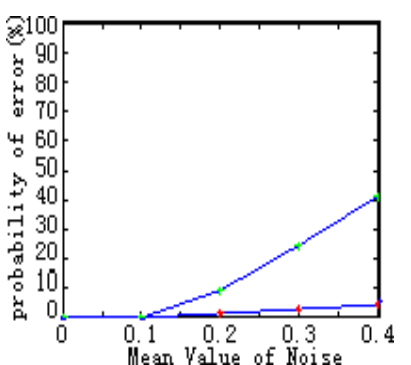

(b) 


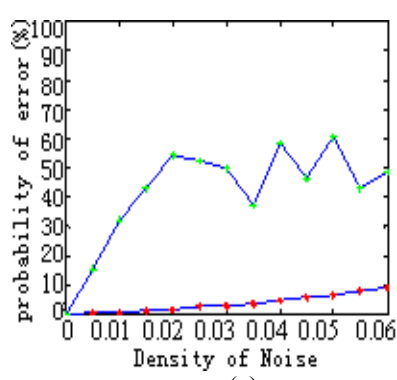

(c)

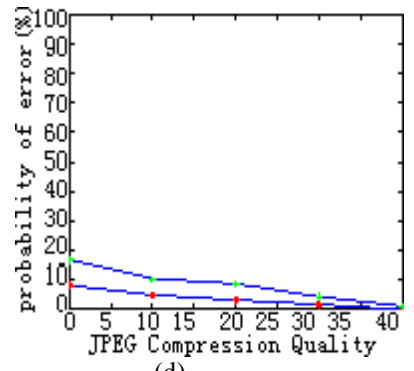

(d)
Figure 4. Experiment result: (a) scaling experiment (b) Gaussian noise experiment (c) salt and pepper noise experiment (d) JPEG compression experiment

\section{CONCLUSIONS}

This paper proposes a novel watermarking algorithm for binary images with robustness. Without reducing the invisibility of the images, the proposed method based on Haar Wavelet Transform can find more flippable pixels than common method. It is the key to improve the capacity of the binary images. With the help of dynamic step size, the watermarking can be against scaling attacks, noise attacks and JPEG compression. The experiment confirms that the method is practical and advantage. With the development of multimedia and Internet, more and more digital documents are spread over for a wide range of applications, such as government documents, business contracts and certificates, etc. The algorithm can be applied to detect unauthorized or protect copyright use of watermarked binary pictures, documents and signatures, such as copy, alteration, scanning and printing.

\section{REFERENCES}

[1] M. Wu, and B. Liu, Data Hiding in Binary Image for Authentication and Annotation, IEEE Transactions on Multimedia, IEEE, April 2004: 528-538.

[2] Muharemagic, and E. Furht, An Innovative Pixel Scoring Method for Watermarking of Binary Document Images, IEEE International Conference on Multimedia and Expo, Toronto, Oct. 2006:1589-1592.

[3] O. J. Kwon and W. G. Kim, Binary Wavelet Transform Based method for finding flippable pixels in binary images, Optical Engineering, vol. 47, no. 7, July. 2008.

[4] H. Yang, A. C. Kot, and S. Rahardja, Orthogonal data embedding for binary images in morphological transform domain - a high-capacity approach, IEEE Trans. Multimedia, vol. 10, no. 3, April. 2008: 339351 .

[5] M. G. Lee, and C. S. Avila, Watermarking Based Document Authentication in Script Format, 52nd IEEE International Midwest Symposium on Circuits and Systems, IEEE, Mexico, August 2009: 837-841.

[6] H. Tirandaz, and R. Davarzani, Invisible and High Capacity Data Hiding in Binary Text Images Based on Use of Edge Pixels, 2009 International Conference on Signal Processing Systems, IEEE Computer Society, Singapore, May 2009: 130-134.

[7] B. He, and Y. Wu, A Robust Binary Text Digital Watermarking Algorithm for Print-scan Process, World Congress on Computer Science and Information Engineering, IEEE Computer Society, America, July 2009: 290-294.

[8] X. Zhou, W. Zhao, and Z. Wang, Security Theory and Attack Analysis for Text Watermarking, International conference on Ebusiness and information system security, China, May 2009: 1-6.

[9] F.K.-P. Chan, A. W. -C. Fu, C. Yu, Haar wavelets for efficient similarity search of time-series: with and without time warping, IEEE Trans. On Knowledge and Data Engineering. Vol. May. 2003: 686705.

[10] M. M. Mokji, S. A. R. Abu Bakar, Gray Level CO-Occurrence Matrix Computation Based on Haar Wavelet, Computer Graphica, Imaging and Visualisation, Bangkok Thailand, Aug. 2007: 272-279. 